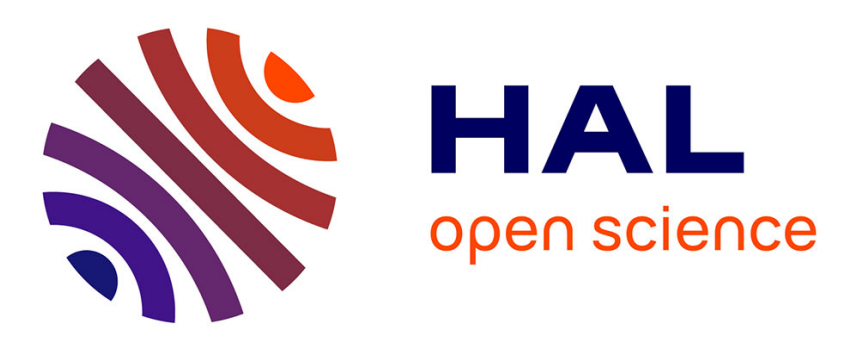

\title{
Is the non-observation of neutrinoless double beta decay a question of sensitivity? \\ Ung Chan Tsan
}

\section{To cite this version:}

Ung Chan Tsan. Is the non-observation of neutrinoless double beta decay a question of sensitivity?. International Journal of Modern Physics E, 2003, 12, pp.439-448. 10.1142/S0218301303001405 . in2p3-00013697

\section{HAL Id: in2p3-00013697 https://hal.in2p3.fr/in2p3-00013697}

Submitted on 30 Oct 2003

HAL is a multi-disciplinary open access archive for the deposit and dissemination of scientific research documents, whether they are published or not. The documents may come from teaching and research institutions in France or abroad, or from public or private research centers.
L'archive ouverte pluridisciplinaire HAL, est destinée au dépôt et à la diffusion de documents scientifiques de niveau recherche, publiés ou non, émanant des établissements d'enseignement et de recherche français ou étrangers, des laboratoires publics ou privés. 
IS THE NON OBSERVATION OF NEUTRINOLESS DOUBLE BETA DECAY A QUESTION OF SENSITIVITY?

\author{
TSAN UNG CHAN \\ Institut des Sciences Nucléaires, IN2P3-CNRS et Université Joseph Fourier \\ 53 Avenue des Martyrs, F38026 Grenoble Cedex FRANCE
}

The hypothetical neutrinoless double beta decay is possible only if the neutrino is a truly neutral particle and if it is massive. A truly neutral particle (e.g. a particle identical with its antiparticle) should have all its algebraic intrinsic properties equal to zero, in particular, its lepton number should be 0 . Now, since the neutrino is a lepton, its lepton number should be 1 . This contradiction would lead to conclude that neutrinoless double beta decay could not take place in nature. This conclusion is, up to now, in agreement with persistent failures to put this long sought hypothetical key decay into evidence despite huge efforts dedicated to this aim. 


\section{Introduction}

Recently, Klapdor-Kleingrothaus et al claimed to have observed evidence for neutrinoless double beta decay with a half-life of around $1.510^{25}$ years (1). This paper gave rise to several reports and comments $(2,3)$. The observation of this long sought decay_if confirmed_would be of immense importance. This sole evidence would immediately infer not only that the neutrino is a Majorana particle (e.g. the neutrino and the antineutrino are the same particle or $v=\bar{v}$ ) but also that its mass is definitely not zero with a value of around $0.39 \mathrm{eV}$. Moreover it would also imply that there exists at least one case where the lepton number $\mathrm{L}$ is not conserved in weak interaction while it is well known that weak interaction as well as other fundamental interactions (strong interaction, electromagnetism) of the Standard Model do conserve L. A list of implications was subsequently and logically deduced, in particular "all models predicting Dirac masses are ruled out and leptogenesis becomes a natural choice" (4). It is striking that so much important consequences could be derived directly from the evidence of this simple key process. So, it is particularly important to be certain that the statement of its existence is solidly founded by providing unambiguous proof. Moreover, we should also remind that all other reactions involving explicitly the neutrino are so far understood uniquely with the opposite assumption: ( $v$ is different from $\bar{v}$ ). Since the neutrino, as any other particle, has to be either identical to or different from its antiparticle, it is necessary to solve this logical contradiction, if it is demonstrated that neutrinoless double beta decay really takes place in nature or if it is considered that it actually exists but is not revealed because of the lack of sensitivity. Curiously, such considerations had been completely overlooked and had not been explicitly discussed and taken into account in works deducing systematically mass limit from lifetime limit. $v$ is different from $\bar{v}$ and $v$ is identical to $\bar{v}$ are two mutually exclusive statements. To be coherent, if we adopt one statement to explain one part of available data we cannot explain the other part of available data with the opposite statement. If neutrinoless double beta decay takes really place through weak interaction then we have to give up to the concept of lepton number and consequently the conservation of lepton number in weak interaction. Conversely, if we admit that the neutrino has $\mathrm{L}=1$, then neutrinoless double beta decay should be absent. Anyway, whatever the adopted statement is, we have to follow our ideas through to their logical conclusions.

\section{Present experimental status of $\beta \beta 0 \mathrm{v}$ decay}

Klapdor-Kleingrothaus et al (1) used a particular mathematical process (the Bayersian method) for low counting rates to deduce the evidence of neutrinoless double beta decay. The correctness of their deduction was immediately questioned. Aalseth et al (5) in a detailed discussion stated that "consideration of these limitations leads to the 
conclusion that there is no basis for the claim presented in the paper". Ferruglio et al (6) expressed a similar doubt ("in conclusion, we do not see a really significant evidence for $0 v 2 \beta$ in published data"). We remark also that, an analysis of the practically same data, by Heidelberg Moscow collaboration (7), led only to an lower limit for the halflife. Sum spectrum presented in (1) corresponding to $54.981 \mathrm{kgy}$ of counting was not fundamentally different from spectrum presented in (7) corresponding to $53.9 \mathrm{kgy}$ of counting. The evidence of $\beta \beta 0 \mathrm{v}$ decay was suggested only by new mathematical process while experimental spectra were almost identical and did not show evidence of peak at the expected energy. Indeed, the experimental status of $\beta \beta 0 \mathrm{v}$ decay studies on several nuclei before the publication of (1) was very well summarized by Fiorini (8): "No evidence, but also not even a hint, has been presented so far for the dreamed peak in the electron sum corresponding to neutrinoless double beta decay ". After scrutinizing the spectra presented in (1), in (7) and in (9), we are convinced that the statement of Fiorini still reflects the present experimental status. There is so far no observed evidence of this process despite heroic experimental efforts dedicated to try to put it into evidence. The conventional approach isolates $\beta \beta 0 \mathrm{v}$ decay from the context of reactions governed by weak interaction, in particular those involving explicitly the neutrino ( $\beta v$ decay, $\beta \beta 2 v$ decay). It indeed admits implicitly that the non observation of this process is uniquely a question of sensitivity. There are then only two alternatives: either $\beta \beta 0 v$ decay turns out to be clearly observed implying thus $v=\bar{v}$, it would then require the complete revision of the Standard Model; or it would be endlessly no evidence of this process and a smaller and smaller upper limit of Majorana mass would be endlessly deduced from the greater and greater lower limit of the experimentally observed lifetime.

This standpoint is however perplexing: it means that whatever the outcome is (observation or non observation of $\beta \beta 0 v$ decay), the neutrino should be a Majorana particle with a tiny mass (definite if $\beta \beta 0 v$ decay is finally observed, and with an upper limit if this decay continues to be not observed) while the Standard Model accounts for all other known reactions involving $v$ with the opposite assumption. But, we must realize that if the observation of this process infers that the neutrino is necessarily a Majorana particle with a finite mass, the interpretation of the non observation is not univocal as suggested by this traditional point of view which leaves indeed the possibility that the neutrino is not a Majorana particle in the dark. If the neutrino is not a Majorana particle, $\beta \beta 0 \mathrm{v}$ decay does not take place in nature and thus can never be observed. Persistent failures to detect this process may reflect in reality the evidence of its absence. Unfortunately, from the sole non observation of $\beta \beta 0 \mathrm{v}$ decay, we can not decide which is the correct choice. The situation is, in this case, endlessly undecidable: the neutrino may be a Majorana particle with a non detectable mass or it may be a Dirac particle with a detectable mass or a non detectable mass. 


\section{Concept of true neutrality}

To stick only to $\beta \beta 0 v$ decay would thus lead to an endlessly undecidable situation, unless unexpectedly future experiments will give an univocal proof of its existence. One possible way to get out of this embarrassing situation could be to come back to the very definition of a particle identical with its own antiparticle and to examine the context in which $\beta \beta 0 v$ decay was proposed to recognize whether the neutrino is distinct or indiscernable from the antineutrino.

The concept of true neutrality was first introduced by Majorana (10) who raised explicitly the following question about the two then known neutral fermions, neutron and neutrino: are they identical with their own antiparticle? A charged particle is different from its antiparticle because their charges are different but an electrically neutral particle is either identical with its antiparticle (a Majorana particle or a Truly Neutral Particle (TNP)) or different from its antiparticle (a Not Truly Neutral Particle (NTNP)).

It is essential to explicit the meaning of two identical particles. It is natural and logical to state that two particles are identical if and only if there is no means to distinguish them by their intrinsic properties or put in another way if all their intrinsic properties are equal. A TNP has to be an electrically neutral particle. But conversely, an electrically neutral particle is not necessarily a TNP. It is only potentially a TNP. A particle can be its own antiparticle (can be a TNP), only if, as far as we know, all known properties are identical for the particle and its antiparticle. And moreover, to be complete, every time that a new property of a particle is discovered, it should be the same for its antiparticle. Properties which are meaningful only for positive values (such as mass, half-life, spin which are positive quantities) are the same for a particle and its antiparticle. But properties which are meaningful for positive, negative and null values (such as Q, magnetic moment, baryon number A, lepton number L, flavours ... which are algebraic quantities) are opposite for a particle and its antiparticle. The immediate consequence is: if any property of this latter class is different from zero, the neutral particle in question is not a TNP.

\section{Strategy of Racah to show that the neutron is not a truly neutral particle}

Majorana put forward a new theory valid for the neutron and the neutrino if they were identical to their own antiparticles. He did not suggest arguments or experiments to decide between his new theory valid only if the neutral particle in question is indiscernable from its antiparticle or a simple extension of the Dirac equation to neutral particles. Indeed in the 1930s, it was known that the magnetic moment of the neutron is different from zero. Racah (11) immediately pointed out that this sole difference was sufficient to infer that the neutron and the antineutron are distinct (another argument leading to the same conclusion is that the neutron only decays into $\mathrm{p}+\mathrm{e}^{-}+\bar{v}$ channel). 
The neutron is not a TNP since a TNP has necessarily a strictly null magnetic moment. We note that if a neutral particle $\mathrm{N}$ decays both into a channel and its conjugate channel, $\mathrm{N}$ is a TNP. So, if the neutron decays also into the conjugate channel, it would imply that the neutron is a TNP. The absence of the conjugate channel is perfectly consistent with the fact that the neutron and the antineutron are different. There would be inconsistency if the neutron with non zero magnetic moment could decay into the conjugate channel. At that time, one ignored that besides electric charge $\mathrm{Q}$, particles are also characterized by a set of additive quantum numbers such as A, L, flavours, colours ..., otherwise Racah could have drawn the same conclusion by remarking for example that the baryon number A of the neutron is not zero. Antineutron was discovered in 1956.

The reasoning of Racah did not allow to show that the neutrino is different from the antineutrino, because there was no evidence that its magnetic moment was different from zero. In other words, the neutrino remains potentially a TNP while the neutron is clearly not a TNP. We remark that, even now, more than sixty years later, despite considerable improvements of sensitivity, experiments have not excluded the zero value. The limit of the magnetic moment of the neutrino is smaller and smaller and is now $1.510^{-10} \mu_{\mathrm{B}}(12)$. The nature of the neutrino (TNP or NTNP) remains an open question as long as its magnetic moment can be zero. A TNP has necessarily a null magnetic moment but it is not impossible that a NTNP has also a null magnetic moment. If we restrict our considerations only to $\mathrm{Q}$ and magnetic moment, we can conclude with certainty that the neutron is distinct from its antiparticle (many experimental facts have subsequently confirmed this statement), while the case of the neutrino is undecidable. But we know that it has to be either TNP or NTNP. In the 1930 s, one ignored that any particle (and in particular the neutrino) is characterized by properties other than Q and magnetic moment. It is then paradoxal to continue to reason as at the time of Furry by sticking only to $\beta \beta$ decay and not to use our present knowledge on properties of the neutrino that were unknown in the 1930s, to try to settle the issue. Maybe, these properties could reveal the nature of the neutrino.

\section{5. $\beta \beta$ decay, Davis experiment and the nature of the neutrino}

Many experimental and theoretical breakthroughs were accomplished in the 1930s: concept of antiparticle, discoveries of positron and neutron, concept of the neutrino by Pauli in order to explain the puzzling $\beta$ spectra which instead to be discrete were continuous. Fermi incorporated the hypothetical neutrino in his theory of beta decay to account for numerous experimental results. In 1935, Goeppert-Meyer (13) explained that some even-even nuclei are only apparently stable because in reality they could decay through a postulated process $\beta \beta$ decay. Her calculations based on Fermi theory (second order process) showed that the expected lifetimes of this process were 
exceedingly slow even on a geologic time scale and were out of reach of experiments of that time.

It was in this context that, triggered by Majorana's ideas, in 1939, Furry (14) realized that within the framework of the knowledge of that time, $\beta \beta$ decay could give a firm answer to the nature of the neutrino. Basically, beta decay transforms a neutron into three particles: proton, electron and antineutrino. And $\beta \beta$ decay transforms two neutrons of a parent nuclei of atomic number $Z$ into a daughter nuclei with $Z+2$, two electrons and two antineutrinos. For example:

$76_{\mathrm{Ge}-->76} \mathrm{Se}+2 \mathrm{e}^{-}+2 \bar{v}$

It is clear that $\mathrm{Q}$ is conserved in this so-called $\beta \beta 2 v$ process, whether the neutrino is TNP or NTNP since in any case $Q(v)=Q(\bar{v} \quad)=0$. In other words, $\beta \beta 2 v$ is always an allowed process whatever the nature of the neutrino is, if the conservation of $\mathrm{Q}$ is the only conservation law to be respected (it was the only known material conservation law of that time). But, if we assume now moreover that $v=\bar{v}$, then another additional process is possible, the so-called $\beta \beta 0 \mathrm{v}$ process where no neutrino is emitted, the first neutrino emitted by the first neutron being absorbed by the second neutron giving at last only two electrons. In our example, it corresponds to the following reaction: ${ }^{76} \mathrm{Ge}-->76 \mathrm{Se}+2 \mathrm{e}^{-}$.

The most interesting feature is that $\beta \beta 0 v$ decay should be enhanced by a very huge phase factor of many orders of magnitude over the rate of $\beta \beta 2 v$ decay. At that time, only geochemical methods were used and they did not distinguish $\beta \beta 0 v$ from $\beta \beta 2 v$, the two processes giving the same daughter nuclei. But the great difference of lifetime between the two processes should be a powerful means to provide a clear-cut answer to the nature of the neutrino. As soon as sensitivity was sufficient, if $\beta \beta$ decay were detected (relative to $\beta \beta 0 v$, the contribution of $\beta \beta 2 v$ should be completely negligible), then the neutrino is a Majorana particle or in other word $v=\bar{v}$; if $\beta \beta$ were not detected then the neutrino is a Dirac particle, i.e. is different from its antiparticle.

So, curiously, without knowing anything else about the properties of the neutrino, in particular, ignoring that several other quantum numbers (A, L, flavours...) are indeed associated to particles, it was possible to determine unambiguously the nature of the neutrino.

_if the magnetic moment of the neutrino is not zero, $v$ is different from $\bar{v}$ (same reasoning as Racah (11) on neutron)

_if $\beta \beta 0 v$ exists, $v$ is identical with $\bar{v}$, implying then logically that all known and yet unknown properties should be the same for $v$ and $\bar{v}$.

In 1955, Davis (15) showed that the reaction $\bar{v}+37 \mathrm{Cl}-->\mathrm{e}^{-}+37 \mathrm{Ar}$ did not occur (strictly speaking, no evidence of this process), favouring then the hypothesis of 
the difference between $v$ and $\bar{v}$. And one did believe that the Davis experiment proved that the neutrino is a Dirac particle. This belief was in agreement with the non observation of $\beta \beta$ decay in all then performed experiments. But this interpretation was outdated after the overthrow of parity conservation in $\beta$-decay $(16,17)$. It was indeed realized that because of helicity, since the neutrino is assumed to be massless, $\bar{v}+$ ${ }^{37} \mathrm{Cl}-->\mathrm{e}^{-}+{ }^{37} \mathrm{Ar}$ and $\beta \beta 0 \mathrm{v}$ decay were not possible regardless of whether it was a Majorana particle or not. The impossibility of changing the helicity of the neutrino which was believed to have zero mass, was sufficient to explain naturally the absence of Davis process and $\beta \beta 0 v$ process. In other words, these processes, imagined to recognize the nature of the neutrino (presence means Majorana or TNP, absence means Dirac or NTNP), had indeed to be always absent. And the non observation of theses processes in experiments dedicated to this aim was indeed the consequence of massless neutrino. However, we should remark that this argument does not imply that the null result of Davis experiment is not (also) due to the Dirac character of neutrinos. Later, it was realized that massless neutrino is only a theoretical postulate. This question was then re-examined in the hypothesis of a tiny but not null mass. $\beta \beta 0 \mathrm{v}$ decay was again theoretically possible if $v=\bar{v}$, with the additional condition that neutrinos were not massless. $\beta \beta 0 v$ decay became a test for neutrino mass as well as for Majorana neutrinos. (the lifetime of $\beta \beta 0 \mathrm{v}$, if it exists, is believed now to be also linked to the mass of the neutrino and its lifetime tends to infinity when the mass tends to zero. Even if $v=\bar{v}, \beta \beta 0 v$ decay is strictly forbidden if $m_{v}=0$ due to the impossibility to change helicity.)

\section{6. $\beta$ decay and $\beta \beta$ decay}

The present experimental situation about $\beta \beta$ decay strangely looks like the situation of $\beta$ decay: continuous spectra without any hint of a peak at the maximum energy corresponding to no neutrino emission. In $\beta$ decay, a peak at maximum energy would mean that reaction such as $n-->p+e^{-}$is possible and thus it is possible to violate $\mathrm{L}$ (material conservation) and angular momentum (motional conservation) while it is believed that angular momentum should be conserved in all interactions. The absence of peak together with continuous spectra prompted then Pauli to introduce the concept of neutrino. $\beta$ decay is characterized by continuous spectra and absence of peak at maximum energy. The introduction of $v$ naturally explains these two experimental observations. Continuous spectra is the telltale signature of an extra neutral particle $v$ and the absence of peak at the $\mathrm{Q} \beta$ value reflects the absence of beta decay without neutrino.

There is a great analogy between the spectra of $\beta$ decay and $\beta \beta$ decay: both spectra are continuous and no peak has been seen at maximum energy (no neutrino emission) neither in $\beta$ decay nor in $\beta \beta$ decay spectra. $v v$ plays the same role in $\beta \beta$ 
decay as $v$ in $\beta$ decay. The great difference is that $\beta$ decay spectra has much greater statistics than $\beta \beta$ decay due to the huge difference of lifetimes (typically 20 orders of magnitude). However, the absence of peak is not attributed to the same cause. In $\beta$ decay, there is no peak because it would violate angular momentum conservation which has to be respected in all interactions. This process is also forbidden by L conservation. The consequence of the existence of a peak in $\beta$ decay at the $Q \beta$ value would be the breakdown of angular momentum conservation and L conservation. Contrary to the case of $\beta$ decay without neutrino which is forbidden both by angular momentum conservation and $\mathrm{L}$ conservation, $\beta \beta$ without neutrino is not forbidden by angular momentum conservation, apparently it is only forbidden by material type conservation, namely by $\mathrm{L}$ conservation, or viewed from another standpoint, by the fact that the neutrino is different from the antineutrino $\left(\right.$ and/or $\left.m_{V}=0\right)$. $\beta \beta 0 v$ peak would violate $\mathrm{L}$ conservation in a very specific way. The consequence of the existence of $\beta \beta 0 \mathrm{v}$ decay would be not only $v=\bar{v}$, it means also $m_{v}>0$ (properties of the neutrino) and the violation of $\mathrm{L}$ with $\Delta \mathrm{L}=2$, associated to the strict conservation of baryon number $\mathrm{A}$ $(\Delta \mathrm{A}=0)$ (violation of well-known conservation law of weak interaction). However, the non observation of $\beta \beta 0 \mathrm{v}$ decay is again and again a stubborn experimental fact which could be interpreted as a mounting evidence of its real absence. No experimental results require that the neutrino is a Majorana particle. This hypothesis is mainly suggested by theories.

Our knowledge on particle physics and on interactions is now considerably better than that of the time of Majorana and Furry. Now, we know that A, L, flavours are intrinsic properties of particles and Q, A, L and flavours are intimately linked through the extended Gell-Mann and Nishijima formula $(18,19)$. And the experimental situation is also quite clear: $\beta \beta$ decay proceeds through $\beta \beta 2 v$ decay (testimonied by continuous $\beta \beta$ spectra) without any hint of $\beta \beta 0 v$ decay (testimonied by the absence of peak at maximum energy). Intrinsic properties of a particle should be the same whether it is involved implicitly or it is involved explicitly in a reaction. And reactions governed by the same fundamental interaction (strong interaction, electromagnetism or weak interaction) should obey the same specific conservation laws. Otherway, there would be inconsistency.

Unless it turns out that $\beta \beta 0 v$ decay is univocally observed in the future implying then that the neutrino is a TNP, if we stick only to this process (and as long as the magnetic moment of the neutrino is possibly null), the neutrino remains potentially a TNP since it is always possible to suppose that the non observation of $\beta \beta 0 \mathrm{v}$ decay is only a question of sensitivity. But if we realize that this non observation could also simply reflect a real absence then it becomes paradoxal not to consider the possibility that the neutrino is not a TNP by examining intrinsic properties of the neutrino known 
at the present time and yet unknown at the time of Majorana, Racah and Furry. We immediately see that the neutrino is different from the antineutrino by at least one property ( $\mathrm{L}=1$ for the neutrino and $\mathrm{L}=-1$ for the antineutrino). Indeed, $v$ and $\bar{v}$ are also different by opposite flavours. So, $\beta \beta 0 v$ decay must be an impossible process since $v=$ $\bar{v}$ is a necessary condition for the occurrence of $\beta \beta 0 v$ decay. We could predict consequently that whatever the improvement of sensitivity is, the only possibility is to obtain a greater and greater value for lower limit of the lifetime of $\beta \beta 0 v$ decay without any unequivocal positive signal. Future results of Moscow Heidelberg collaboration (20), IGEX collaboration (9,21), NEMO collaboration (22) and may be GENIUS (23) or CUORE (24) will provide interesting confrontation. By contrast, $\beta \beta 2 v$ decay to $0^{+}$ excited state is a possible process (25), although much rarer than that to ground state. Like in the case of $100 \mathrm{Mo}, \beta \beta 2 v$ decay to excited state in other nuclei where $\beta \beta 2 v$ decay to the ground state had been already observed, would be observed provided a sufficient improvement of sensitivity. The mass of the neutrino cannot be deduced from $\beta \beta 0 v$ decay. Its mass has to be measured by another kinds of experiments. From the tritium $\beta$ spectrum, an upper limit of $2.8 \mathrm{eV}$ on the neutrino mass was derived (26).

It is generally admitted that the only way to reveal the Majorana nature of the neutrino (if it is) is to search for $\beta \beta 0 v$ decay. And curiously, the occurrence of $\beta \beta 0 v$ decay would bring much more: the mass of the neutrino would be determined with precision and the conservation of $\mathrm{L}$ would break down in weak interaction. The problem is that up to now, there is no hint of its existence. Maybe, non observation means in reality absence of this decay which does not take place in nature.

\section{Conclusion}

Historically, $\beta \beta 0 \mathrm{v}$ decay was proposed to recognize whether the neutrino is identical to the antineutrino or is different from the antineutrino. Now, in some sense, its existence became a theoretical prejudice implying that the neutrino and the antineutrino are the same particle. However, this interesting prejudice has not been so far backed by any sound experimental proof.

We remark that the sole existence of $\beta \beta 0 v$ decay apparently would lead to two important consequences: one concerning the properties of the neutrino (the neutrino must be identical with the antineutrino and it must be massive) and another concerning the property of the weak interaction ( $\mathrm{L}$ is violated in a specific way $\Delta \mathrm{L}=2$ ). These two consequences are indeed intimately linked and reflect the inconsistency of the hypothesis of a Majorana neutrino. In effect, the neutrino being a lepton has to have $\mathrm{L}=1$ and a Majorana particle (TNP) has to have $\mathrm{L}=0 . \Delta \mathrm{L}=2$ comes from the fact that we suppose that the neutrino has both $\mathrm{L}=1$ (in the general case) and $\mathrm{L}=0$ in this special case. $\beta \beta 0 v$ decay is an impossible process since it requires two contradictory conditions for the neutrino. Another argument against Majorana nature of the neutrino is: the 
neutrino being sensitive to weak force should have a weak charge different from zero, thus it can not be a TNP.

We point out that all known TNP are bosons: composite bosons like mesons formed by a quark and its antiquark or elementary messenger bosons like $\gamma, Z^{0}, R \bar{R}$. A fermion is always associated to $\mathrm{A} \neq 0$ or $\mathrm{L} \neq 0$ thus cannot be a TNP (18).

We could also associate each particle to a vector $\vec{C}$ representing the particle in an abstract space (27). The electric charge Q could be interpreted as the projection of $\vec{C}$ on a vector $\vec{Q}(0)$. If $\vec{C}=\overrightarrow{0}$, particle and antiparticle are the same particle. If $\vec{C} \neq \overrightarrow{0}$, particle and antiparticle are represented by opposite vectors and are different even if $\mathrm{Q}=0$.

Messengers associated to fundamental forces would explain why they have different ranges and different conservation laws. Infinite range of electromagnetism is due to massless $\gamma$; short range of weak interaction is due to massive messengers $Z$ and $\mathrm{W}$; finite range of strong force is similar to finite range of Van der Vaals force in molecules. Strong force and electromagnetism conserve A, L and individual flavours because $\gamma$ and gluons have all these quantities definite and equal to zero while weak interaction conserves $\mathrm{A}, \mathrm{L}$ and total flavour but does not conserve individual flavours because $\mathrm{W}$ and $\mathrm{Z}$ in weak interaction are characterized by $\mathrm{A}, \mathrm{L}$ and total flavour but not by individual flavours.

Many theories which go beyond the Standard Model predict that the neutrino is identical with the antineutrino. However, they have not been supported by the observation of $\beta \beta 0 \mathrm{v}$ decay events. We remark that, if a massive neutrino could be considered as beyond the Standard Model, a Majorana neutrino is indeed against the Standard Model. Physics is essentially an experimental science, any theory has to be confronted with indisputable experimental results. Presently, all available data seem to show coherently that the neutrino is different from the antineutrino implying that neutrinoless double beta decay is an impossible process. However, search for hypothetical $\beta \beta 0 v$ decay remains a powerful means to falsify this statement. Its existence, if unambiguously proved, would jeopardize the Standard Model in its very foundation and would require its complete revision. Considerable improvement of sensitivity has not allowed to obtain the slightest hint of the existence of $\beta \beta 0 v$ decay but has revealed allowed $\beta \beta 2 v$ decay in several nuclei. Their experimental lifetimes are in agreement with theoretical lifetimes expected from the Standard Model. Mass limit of the neutrino deduced from lower lifetime limit of $\beta \beta 0 v$ decay has no physical sense. It is clear that the Standard Model which accounts for almost all experimental data, is nevertheless not sufficient, for example, it fails to explain the observed matterantimatter asymmetry of our universe. It would be thus necessary to go beyond the Standard Model by trying to put forward theories that respect the known properties of particles and in particular the Dirac nature of the neutrino. We remark that any theory 
beyond the Standard Model should be able to account for all features naturally explainable in the framework of the Standard Model.

\section{References}

1)H.V. Klapdor-Kleingrothaus et al, Mod.Phys.Lett A, 16(2001)2409

2)Edwin Cartlidge, Phys. World, March 2002 p5

3)Edward Witten, Nature, 415(2002)969

4)H.V. Klapdor-Kleingrothaus and U. Sarkar, Mod.Phys.Lett A, 16(2001)2469

5)C.E.Aalseth et al, Preprint hep-ex/202018(2002), Mod.Phys.Lett A, 17(2002)1475

6)Ferruccio Feruglio, Alessandro Strumia and Francesco Vissani, Preprint hep$\mathrm{ph} / 0201291(2002)$

7)H.V. Klapdor-Kleingrothaus et al , Eur.Phys. J.A 12(2001)147

8)Ettore Fiorini, Nucl.Phys.B (Proc. Suppl.) 91(2001)262

9)C.E. Aalseth et al, Phys.RevD65(2002)092007

10)Ettore Majorana, Nuovo Cimento, 14 (1937) 171

11)Giulio Racah, Nuovo Cimento, 14 (1937) 322

12)Review of particle properties, Eur.Phys.J C15(2000)1

13)M.Goeppert-Mayer, Phys.Rev.48 (1935) 512

14)W.H.Furry, Phys.Rev.56 (1939),1184

15)R.Davis Jr., Phys.Rev.97 (1955) 766

16)T.D.Lee and C.N.Yang, Phys.Rev.104 (1956) 254

17)C.S.Wu , E.Ambler, R.W.Hayward, D.D.Hoppes and R.P.Hudson , Phys.Rev.105 (1957) 1413

18)Tsan Ung Chan, Int.J.Mod.Phys.E7(1998) 405

19)Tsan Ung Chan, Int.J.Mod.Phys.E7(1998) 747

20)L.Baudis et al, Phys.Lett.B407 (1997) 219

21)C.E.Aalseth et al, Phys.Rev.C59 (1999) 2108 
22)R.Arnold et al, Z.Phys.C72(1996)239

23)J.Hellmig, H.V.Klapdor-Kleingrothaus, Z.Phys.A359(1997)351

24)E.Fiorini, Phys.Rep. 307(1998)309

25)L. De Braeckeleer, M. Hornish, A. Barabash and V. Umatov, Phys.Rev.Lett.86 (2001)3510

26)Ch.Weinheimer, B.Degenddag, A.Bleile, J.Bonn, L.Bornschein, O.Kazachenko, A.Kovalik, E.W.Otten, Phys.Lett.B460 (1999) 219

27)Tsan Ung Chan, Int.J.Mod.Phys.E10(2001) 353 